\title{
Constraints from muon $g-2$ and LFV processes in the Higgs Triplet Model
}

\author{
Takeshi Fukuyama* and Hiroaki Sugiyamat \\ Department of Physics and R-GIRO, \\ Ritsumeikan University, Kusatsu, Shiga, 525-8577, Japan \\ Koji Tsumurat \\ The Abdus Salam ICTP of UNESCO and IAEA, \\ Strada Costiera 11, 34151 Trieste, Italy
}

\begin{abstract}
Constraints from the muon anomalous magnetic dipole moment and lepton flavor violating processes are translated into lower bounds on $v_{\Delta} m_{H^{ \pm \pm}}$in the Higgs Triplet Model by considering correlations through the neutrino mass matrix. The discrepancy of the sign of the contribution to the muon anomalous magnetic dipole moment between the measurement and the prediction in the model is clarified. It is shown that $\mu \rightarrow e \gamma, \tau$ decays (especially, $\tau \rightarrow \bar{\mu} e e$ ), and the muonium conversion can give a more stringent bound on $v_{\Delta} m_{H^{ \pm \pm}}$than the bound from $\mu \rightarrow \bar{e} e e$ which is expected naively to give the most stringent one.
\end{abstract}

PACS numbers: 13.35.-r, 12.60.Fr, 14.60.Pq, 14.80.Cp

\footnotetext{
*Electronic address: fukuyama@se.ritsumei.ac.jp

${ }^{\dagger}$ Electronic address: hiroaki@fc.ritsumei.ac.jp

${ }^{\ddagger}$ Electronic address: ktsumura@ictp.it
} 


\section{INTRODUCTION}

In the standard model of the particle physics (SM), neutrinos are massless particles due to the absence of right-handed neutrinos $\nu_{R}$. The simplest way to give masses to three neutrinos is to add three $\nu_{R}$ similarly to other fermions, which corresponds to six additional particles (three $\nu_{R}$ and three $\overline{\nu_{R}}$ ) to the SM. In the Higgs triplet model (HTM) [1, 2] which we deal with in this article, a complex $S U(2)_{L}$ triplet scalar with the hypercharge $Y=2$ is introduced to the SM in order to have neutrino masses. This model can be regarded as one of the simplest extension of the SM because the number of new particles is six in this model also.

The triplet Higgs boson field with hypercharge $Y=2$ can be parameterized by

$$
\Delta \equiv\left(\begin{array}{cc}
\Delta^{+} / \sqrt{2} & \Delta^{++} \\
\frac{v_{\Delta}}{\sqrt{2}}+\Delta^{0} & -\Delta^{+} / \sqrt{2}
\end{array}\right),
$$

where $v_{\Delta}$ is the vacuum expectation value (VEV) of the triplet Higgs boson. The constraint on the rho parameter, $\rho_{0}=1.0004_{-0.0007}^{+0.0027}$ at $2 \sigma \mathrm{CL}$ (page 137 of [3]), gives an upper limit $v_{\Delta} / v \lesssim 0.01$ where $v=246 \mathrm{GeV}$ is the VEV of the doublet Higgs field, which corresponds to $v_{\Delta} \lesssim 3 \mathrm{GeV}$. There is no stringent bound from quark sector on triplet Higgs bosons because they do not couple to quarks. The interaction of the Higgs triplet with lepton doublets $L_{\ell} \equiv\left(\nu_{\ell L}, \ell_{L}\right)^{T}(\ell=e, \mu, \tau)$ is given by

$$
\mathcal{L}_{\text {triplet-Yukawa }}=h_{\ell \ell^{\prime}} \overline{L_{\ell}^{c}} i \tau_{2} \Delta L_{\ell^{\prime}}+\text { H. c. }
$$

The symmetric matrix $h_{\ell \ell^{\prime}}$ is coupling strength, $\tau_{i}(i=1-3)$ denote the Pauli matrices, and $\overline{L_{\ell}^{c}} \equiv\left(\nu_{\ell L}^{T} C, \ell_{L}^{T} C\right)$ with the charge conjugation operator $C$.

The coupling $h_{\ell \ell^{\prime}}$ has a direct relation to the neutrino mass matrix $m_{\ell \ell^{\prime}}$ in the flavor basis through $v_{\Delta}$ as

$$
h_{\ell \ell^{\prime}}=\frac{1}{\sqrt{2} v_{\Delta}}\left(U_{\mathrm{MNS}}^{*} \operatorname{diag}\left(m_{1}, m_{2} e^{-i \varphi_{1}}, m_{3} e^{-i \varphi_{2}}\right) U_{\mathrm{MNS}}^{\dagger}\right)_{\ell \ell^{\prime}} \equiv \frac{1}{\sqrt{2} v_{\Delta}} m_{\ell \ell^{\prime}} .
$$

The mass eigenvalues $m_{i}$ are taken to be real positive values. We define $\Delta m_{i j}^{2} \equiv m_{i}^{2}-m_{j}^{2}$ and refer to the case of $\Delta m_{31}^{2}>0\left(\Delta m_{31}^{2}<0\right)$ as the normal (inverted) mass ordering. Here neutrinos are required to be Majorana particles ${ }^{1}$, and $\varphi_{1}$ and $\varphi_{2}$ are the Majorana

\footnotetext{
1 In general, even if the Higgs triplet exists, neutrinos can be Dirac particles by adding $\nu_{R}$ also and requiring lepton number conservation which results in $v_{\Delta}=0$. In the HTM we use, neutrino masses are assumed to be given solely by $v_{\Delta}$ and neutrinos are Majorana particles by definition.
} 
phases [2, 4] defined in an interval of $[0,2 \pi)$. The Maki-Nakagawa-Sakata matrix [5] of the neutrino mixing ${ }^{2}$ is parameterized as

$$
U_{\mathrm{MNS}} \equiv\left(\begin{array}{ccc}
c_{12} c_{13} & s_{12} c_{13} & s_{13} e^{-i \delta} \\
-s_{12} c_{23}-c_{12} s_{23} s_{13} e^{i \delta} & c_{12} c_{23}-s_{12} s_{23} s_{13} e^{i \delta} & s_{23} c_{13} \\
s_{12} s_{23}-c_{12} c_{23} s_{13} e^{i \delta} & -c_{12} s_{23}-s_{12} c_{23} s_{13} e^{i \delta} & c_{23} c_{13}
\end{array}\right)
$$

where $s_{i j} \equiv \sin \theta_{i j}$ and $c_{i j} \equiv \cos \theta_{i j}$, and $\delta$ is the Dirac phase. The ranges are chosen as $0 \leq \theta_{i j} \leq \pi / 2$ and $0 \leq \delta<2 \pi$. According to current constraints from neutrino oscillation experiments [7, 8, 9, 10], we use the following values in this article

$$
\begin{aligned}
& \Delta m_{21}^{2}=7.6 \times 10^{-5} \mathrm{eV}^{2}, \quad\left|\Delta m_{31}^{2}\right|=2.4 \times 10^{-3} \mathrm{eV}^{2} \\
& \sin ^{2} 2 \theta_{12}=0.87, \quad \sin ^{2} 2 \theta_{23}=1, \\
& \sin ^{2} 2 \theta_{13}<0.14
\end{aligned}
$$

The absolute scale of the neutrino mass is constrained by tritium beta decay measurements as $m_{\nu} \leq 2.3 \mathrm{eV}(95 \% \mathrm{CL})$ [11] and by cosmological observations as $\sum m_{i}<0.61 \mathrm{eV}$ (95\% CL) or $\sum m_{i}<1.3 \mathrm{eV}$ (WMAP only, 95\% CL) [12].

The HTM has seven physical Higgs bosons which are two CP-even neutral bosons $h^{0}$ (lighter) and $H^{0}$ (heavier), a CP-odd neutral one $A^{0}$, a pair of singly charged bosons $H^{ \pm}$, and a pair of doubly charged bosons $H^{ \pm \pm}$. These Higgs bosons contribute to many lepton flavor violating (LFV) processes. Experimental searches for $\mu \rightarrow \bar{e} e e$ etc. put upper bounds on $\left|h_{\mu e}\right|\left|h_{e e}\right| / m_{H^{ \pm \pm}}^{2}$ etc. (See e.g. [13]), where $m_{H^{ \pm \pm}}$is the mass of $H^{ \pm \pm}$. The couplings $h_{\ell \ell^{\prime}}$ are, however, not free in the HTM because they relate directly to the neutrino mass matrix $m_{\ell \ell^{\prime}}$ as shown in (3). Previous works for dependences of LFV processes on the parameters in $m_{\ell \ell^{\prime}}$ can be found in [6, 14, 15]. In this article, we consider in detail the correlation of upper bounds on $\left|h_{i j}^{*} h_{k l}\right| / m_{H_{ \pm \pm}}^{2}$ from new physics searches and deal with them as lower bounds on $v_{\Delta} m_{H^{ \pm \pm}}$.

$\overline{2}$ We took the definition of the mixing $\nu_{\ell}=\sum_{i} U_{\ell i} \nu_{i}$ according to page 517 of [3] although another definition $\nu_{\ell}=\sum_{i} U_{\ell i}^{*} \nu_{i}$ is used for example, in [6] and on page 163 of [3]. In latter definition, we need to take complex conjugate in the middle equation of (3). 


\section{LOWER BOUND ON $v_{\Delta} m_{H^{ \pm \pm}}$}

\section{A. Constraint from the Muon Anomalous Magnetic Dipole Moment}

Let us consider first the anomalous magnetic dipole moment (MDM) of muon, $a_{\mu} \equiv$ $(g-2) / 2$. The muon anomalous MDM has been measured very precisely [16] as

$$
a_{\mu}^{\exp }=11659208.0(6.3) \times 10^{-10},
$$

where the number in parentheses shows $1 \sigma$ uncertainty. On the other hand, the SM predicts

$$
\begin{aligned}
a_{\mu}^{\mathrm{SM}}[\tau] & =11659193.2(5.2) \times 10^{-10}, \\
a_{\mu}^{\mathrm{SM}}\left[e^{+} e^{-}\right] & =11659177.7(5.1) \times 10^{-10},
\end{aligned}
$$

where the hadronic contributions to $a_{\mu}^{\mathrm{SM}}[\tau]$ and $a_{\mu}^{\mathrm{SM}}\left[e^{+} e^{-}\right]$were calculated [17] by using data of hadronic $\tau$ decay and $e^{+} e^{-}$annihilation to hadrons, respectively (See also [18, 19, 20, 21, 22]). The deviations of the SM predictions from the experimental result are given by

$$
\begin{aligned}
\Delta a_{\mu}[\tau] & \equiv a_{\mu}^{\exp }-a_{\mu}^{\mathrm{SM}}[\tau]=14.8(8.2) \times 10^{-10}, \\
\Delta a_{\mu}\left[e^{+} e^{-}\right] & \equiv a_{\mu}^{\exp }-a_{\mu}^{\mathrm{SM}}\left[e^{+} e^{-}\right]=30.3(8.1) \times 10^{-10} .
\end{aligned}
$$

These values of $\Delta a_{\mu}[\tau]$ and $\Delta a_{\mu}\left[e^{+} e^{-}\right]$correspond to $1.8 \sigma$ and $3.7 \sigma$ deviations from SM predictions, respectively.

New contributions to $a_{\mu}$ at the 1-loop level in the HTM come mainly from $H^{ \pm \pm}$and $H^{ \pm}$. The Yukawa interactions of $H^{ \pm}$, which are mixtures of doublet and triplet Higgs bosons, and of $H^{ \pm \pm}\left(=\Delta^{ \pm \pm}\right)$are written by

$$
\mathcal{L}_{\text {triplet-Yukawa }}^{H^{ \pm} H^{ \pm \pm}}=-\sqrt{2} \frac{v}{\sqrt{v^{2}+2 v_{\Delta}^{2}}}\left(U_{\mathrm{MNS}}^{T} h\right)_{i \ell} \overline{\nu_{i}^{c}} P_{L} \ell H^{+}-h_{\ell \ell^{\prime}} \overline{\ell^{c}} P_{L} \ell^{\prime} H^{++}+\text {H.c. },
$$

where $P_{L} \equiv\left(1-\gamma^{5}\right) / 2$ and $\nu_{i}$ represent mass eigenstates of Majorana neutrinos which satisfy conditions $\nu_{i}=\nu_{i}^{c}$. The 1-loop contribution of $H^{ \pm}$through the triplet Yukawa interaction ${ }^{3}$

\footnotetext{
3 The contribution through $m_{\mu} / v$ is ignored because it is suppressed by $v_{\Delta}^{2} / v^{2}$.
} 
is calculated as

$$
\begin{aligned}
a_{\mu}^{H^{ \pm}} & =\frac{m_{\mu}^{2}}{8 \pi^{2} m_{H^{ \pm}}^{2}} \frac{v^{2}}{v^{2}+2 v_{\Delta}^{2}} \sum_{i}\left(h^{\dagger} U_{\mathrm{MNS}}^{*}\right)_{\mu i}\left(U_{\mathrm{MNS}}^{T} h\right)_{i \mu} \\
& \quad \int_{0}^{1} d t \frac{-t^{2}(1-t)}{R_{H^{ \pm}}^{\mu} t^{2}+\left(1-R_{H^{ \pm}}^{\mu}-R_{H^{ \pm}}^{i}\right) t+R_{H^{ \pm}}^{i}} \\
& \simeq-\frac{\left\langle m^{2}\right\rangle_{\mu \mu}}{96 \pi^{2}} \frac{m_{\mu}^{2}}{v_{\Delta}^{2} m_{H^{ \pm}}^{2}},
\end{aligned}
$$

and the $H^{ \pm \pm}$contribution is given by

$$
\begin{aligned}
a_{\mu}^{H^{ \pm \pm}} & =\frac{m_{\mu}^{2}}{8 \pi^{2} m_{H^{ \pm \pm}}^{2}} \sum_{\ell}\left(h^{\dagger}\right)_{\mu \ell} h_{\ell \mu} \int_{0}^{1} d t\left[\frac{-4 t^{2}(1-t)}{R_{H^{ \pm \pm}}^{\mu} t^{2}+\left(1-R_{H^{ \pm \pm}}^{\mu}-R_{H^{ \pm \pm}}^{\ell}\right) t+R_{H^{ \pm \pm}}^{\ell}}\right. \\
& \left.\simeq \frac{-2 t^{2}(1-t)}{R_{H^{ \pm \pm}}^{\mu} t^{2}+\left(R_{H^{ \pm \pm}}^{\ell}-R_{H^{ \pm \pm}}^{\mu}-1\right) t+1}\right] \\
& \simeq-\frac{\left\langle m^{2}\right\rangle_{\mu \mu}}{12 \pi^{2}} \frac{m_{\mu}^{2}}{v_{\Delta}^{2} m_{H^{ \pm \pm}}^{2}} .
\end{aligned}
$$

Here we have defined

$$
\begin{aligned}
R_{b}^{a} & \equiv \frac{m_{a}^{2}}{m_{b}^{2}} \\
\left\langle m^{2}\right\rangle_{\ell \ell^{\prime}} & \equiv\left(U_{\mathrm{MNS}} \operatorname{diag}\left(m_{1}^{2}, m_{2}^{2}, m_{3}^{2}\right) U_{\mathrm{MNS}}^{\dagger}\right)_{\ell \ell^{\prime}}=2 v_{\Delta}^{2}\left(h^{\dagger} h\right)_{\ell \ell^{\prime}}
\end{aligned}
$$

Note that $\left\langle m^{2}\right\rangle_{\ell \ell^{\prime}}$ does not depend on Majorana phases and $\left\langle m^{2}\right\rangle_{\ell \ell}$ is positive definite. Thus $a_{\mu}^{\mathrm{HTM}} \equiv a_{\mu}^{H^{ \pm}}+a_{\mu}^{H^{ \pm \pm}}$is negative definite though $\Delta a_{\mu}$ is positive. The minus sign of the contributions from Higgs triplets has been known [13, 23] but it does not seem to be taken seriously probably because of confusions about the combination $\left(h^{\dagger} h\right)_{\mu \mu}$. Usually the combination seems to be written as $\left(h_{\mu \mu}\right)^{2}$ or $\left(h^{2}\right)_{\mu \mu}$, for which sign of $a_{\mu}^{\mathrm{HTM}}$ can be flipped, and then it seems possible to obtain a (finite) constraint on $h_{\ell \ell^{\prime}}$. Actually, any value of $h_{\ell \ell^{\prime}}$ can not fit $\Delta a_{\mu}\left[e^{+} e^{-}\right]\left(\Delta a_{\mu}[\tau]\right)$ at $3.7 \sigma(1.8 \sigma)$ because of the wrong sign of $a_{\mu}^{\text {HTM }}$.

Concerning only on the sign, the 1-loop contribution from $H^{0}$ can have the right sign to explain $\Delta a_{\mu}$ (see [24] for the case in the type II two Higgs doublet model (2HDM-II)). However, the contribution has a suppression with $v_{\Delta}^{2} / v^{2}$ in the HTM because $\Delta^{0}$ does not couple with charged leptons at the tree level ${ }^{4}$. Although in the Barr-Zee type [25] 2-loop diagrams the right-sign contribution of $A^{0}$ can be important in some models like the 2HDMII [26] and the minimal supersymmetric standard model (MSSM) [27], such a situation does

4 The modification of the contribution to $a_{\mu}^{\mathrm{SM}}$ from $h^{0}$ is also suppressed by $v_{\Delta}^{2} / v^{2}$. 


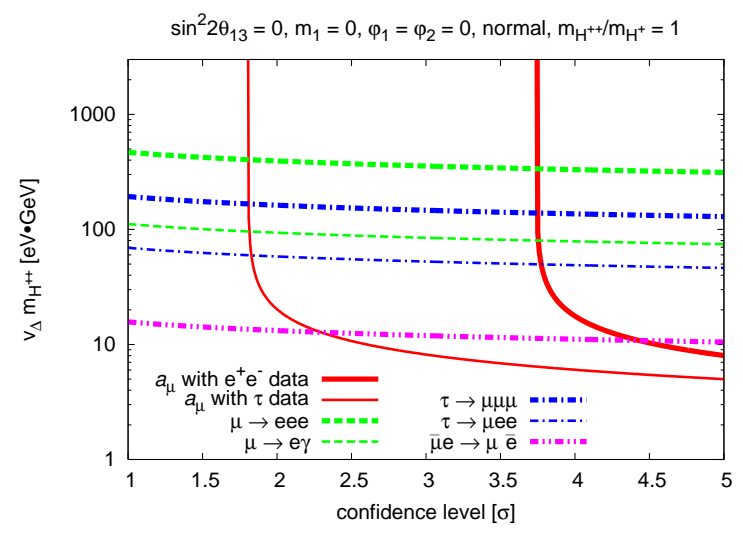

FIG. 1: Lower bounds on $v_{\Delta} m_{H^{ \pm \pm}}$given by constraints on muon anomalous MDM and LFV processes as functions of the confidence level. All parameters in the neutrino mass matrix are fixed as an example (see the text for the values) for the normal mass ordering. We take $m_{H^{ \pm \pm}}=m_{H^{ \pm}}$ for simplicity.

not happen in the HTM because couplings of $A^{0}\left(\simeq \operatorname{Im}\left(\Delta^{0}\right)\right)$ with quarks and charged leptons are also suppressed by $v_{\Delta} / v$.

The definite sign of $a_{\mu}^{\mathrm{HTM}}$ is a feature of the simpleness and the predictability of the HTM. In the MSSM in contrast, the contributions from supersymmetric particles to $a_{\mu}$ can have the right sign easily by the appropriate choice of the sign of the Higgs mass parameter $\mu_{H}$ [28, 29]. As the result, the HTM is somehow disfavored by the muon anomalous MDM and it results in a strong constraint on the model. This is also the case for other models (e.g. the Zee-Babu model [30]) which do not have extra neutral Higgs bosons with sizable couplings to charged leptons similarly to the HTM. Of course, the positive $\Delta a_{\mu}$ does not seem conclusive yet and it does not mean exclusion of the HTM. The difference between $\Delta a_{\mu}\left[e^{+} e^{-}\right]$and $\Delta a_{\mu}[\tau]$ may indicate existence of new physics in the quark sector which is not modified in the HTM.

Hereafter we take $m_{H^{ \pm \pm}}=m_{H^{ \pm}}$for simplicity. The large splitting of their masses is disfavored by the constraint on the $\rho$ parameter. Once we fix the neutrino mass matrix, muon anomalous MDM and LFV processes are interpreted as lower bounds on $v_{\Delta} m_{H^{ \pm \pm}}$. Figure 1 shows the lower bounds with respect to the confidence level in a unit of the standard deviation $\sigma$ and they are given by constraints on the muon anomalous MDM with $e^{+} e^{-}$data (bold solid red line), the MDM with $\tau$ data (solid red line), $\mu \rightarrow \bar{e} e e$ (bold dashed green line), 
$\mu \rightarrow e \gamma$ (dashed green line), $\tau \rightarrow \bar{\mu} \mu \mu$ (bold dash-dotted blue line), $\tau \rightarrow \bar{\mu} e e$ (dash-dotted blue line), and the muonium $\left(\mu^{+} e^{-}\right)$conversion to the anti-muonium (bold dash-dot-dotted magenta line). Bounds from $\tau \rightarrow \bar{\mu} \mu \mu$ and $\tau \rightarrow \bar{\mu} e e$ are important in our analysis among six possible $\tau \rightarrow \bar{\ell} \ell^{\prime} \ell^{\prime \prime}$. Formulae of branching ratios of these LFV decays in the HTM and their current bounds at 90\% CL are

$$
\begin{aligned}
\operatorname{BR}(\mu \rightarrow \bar{e} e e) & =\frac{\left|m_{\mu e}\right|^{2}\left|m_{e e}\right|^{2}}{16 G_{F}^{2} v_{\Delta}^{4} m_{H^{ \pm \pm}}^{4}}<1.0 \times 10^{-12}[31], \\
\operatorname{BR}(\mu \rightarrow e \gamma) & =\frac{27 \alpha\left|\left\langle m^{2}\right\rangle_{e \mu}\right|^{2}}{256 \pi G_{F}^{2} v_{\Delta}^{4} m_{H^{ \pm \pm}}^{4}}<1.2 \times 10^{-11}[32], \\
\operatorname{BR}(\tau \rightarrow \bar{\mu} \mu \mu) & =\frac{\left|m_{\tau \mu}\right|^{2}\left|m_{\mu \mu}\right|^{2}}{16 G_{F}^{2} v_{\Delta}^{4} m_{H^{ \pm \pm}}^{4}} \operatorname{BR}\left(\tau \rightarrow \mu \bar{\nu}_{\mu} \nu_{\tau}\right)<3.2 \times 10^{-8}[33], \\
\operatorname{BR}(\tau \rightarrow \bar{\mu} e e) & =\frac{\left|m_{\tau \mu}\right|^{2}\left|m_{e e}\right|^{2}}{16 G_{F}^{2} v_{\Delta}^{4} m_{H^{ \pm \pm}}^{4}} \operatorname{BR}\left(\tau \rightarrow \mu \bar{\nu}_{\mu} \nu_{\tau}\right)<2.0 \times 10^{-8}[33],
\end{aligned}
$$

where $\operatorname{BR}\left(\tau \rightarrow \mu \bar{\nu}_{\mu} \nu_{\tau}\right)=17 \%, \alpha=1 / 137$ stands for the fine structure constant, and $G_{F}=1.17 \times 10^{-5} \mathrm{GeV}^{-2}$ denotes the Fermi coupling constant. The effective Lagrangian for the muonium conversion is

$$
\mathcal{L}_{M \bar{M}}=2 \sqrt{2} G_{M \bar{M}}\left(\bar{\mu} \gamma^{\rho} P_{L} e\right)\left(\bar{\mu} \gamma_{\rho} P_{L} e\right)=4 \sqrt{2} G_{M \bar{M}}\left(\bar{\mu} P_{R} \mu^{c}\right)\left(\overline{e^{c}} P_{L} e\right)
$$

The formula of the coupling $G_{M \bar{M}}$ in the HTM and current constraint at 90\% CL for that are

$$
\left(\frac{\left|G_{M \bar{M}}\right|}{G_{F}}\right)^{2}=\frac{\left|m_{e e}\right|^{2}\left|m_{\mu \mu}\right|^{2}}{128 G_{F}^{2} v_{\Delta}^{4} m_{H^{ \pm \pm}}^{4}}<\left(3.0 \times 10^{-3}\right)^{2}[34] .
$$

In Fig. 1, parameters of the neutrino mass matrix are fixed by (5), (6), and the following values as an example: $m_{1}=0, \sin ^{2} 2 \theta_{13}=0, \varphi_{1}=\varphi_{2}=0$. With these values of parameters, we have $\left\langle m^{2}\right\rangle_{\mu \mu}=1.2 \times 10^{-3} \mathrm{eV}^{2},\left|m_{\mu e}\right|^{2}\left|m_{e e}\right|^{2}=6.4 \times 10^{-11} \mathrm{eV}^{4},\left|\left\langle m^{2}\right\rangle_{e \mu}\right|^{2}=6.3 \times 10^{-10} \mathrm{eV}^{4}$, $\left|m_{\tau \mu}\right|^{2}\left|m_{\mu \mu}\right|^{2}=3.5 \times 10^{-7} \mathrm{eV}^{4},\left|m_{\tau \mu}\right|^{2}\left|m_{e e}\right|^{2}=3.6 \times 10^{-9} \mathrm{eV}^{4}$, and $\left|m_{e e}\right|^{2}\left|m_{\mu \mu}\right|^{2}=5.9 \times$ $10^{-9} \mathrm{eV}^{4}$. Bounds (20) $-(23)$ and (25) at $90 \% \mathrm{CL}$ are translated naively into $x \sigma$ CL bounds by multiplying $x / 1.64$ because $90 \%$ CL corresponds to $1.64 \sigma$. Below around $1.8 \sigma(3.7 \sigma)$, the muon anomalous MDM $\Delta a_{\mu}[\tau]\left(\Delta a_{\mu}\left[e^{+} e^{-}\right]\right)$gives the strongest constraint on the HTM but it becomes weaker rapidly than other constraints at higher confidence levels. Hereafter, we take $\Delta a_{\mu}[\tau]$ and concentrate ourselves on $2 \sigma$ CL in order to avoid qualitative disagreement with $\Delta a_{\mu}[\tau]$ in the HTM. 

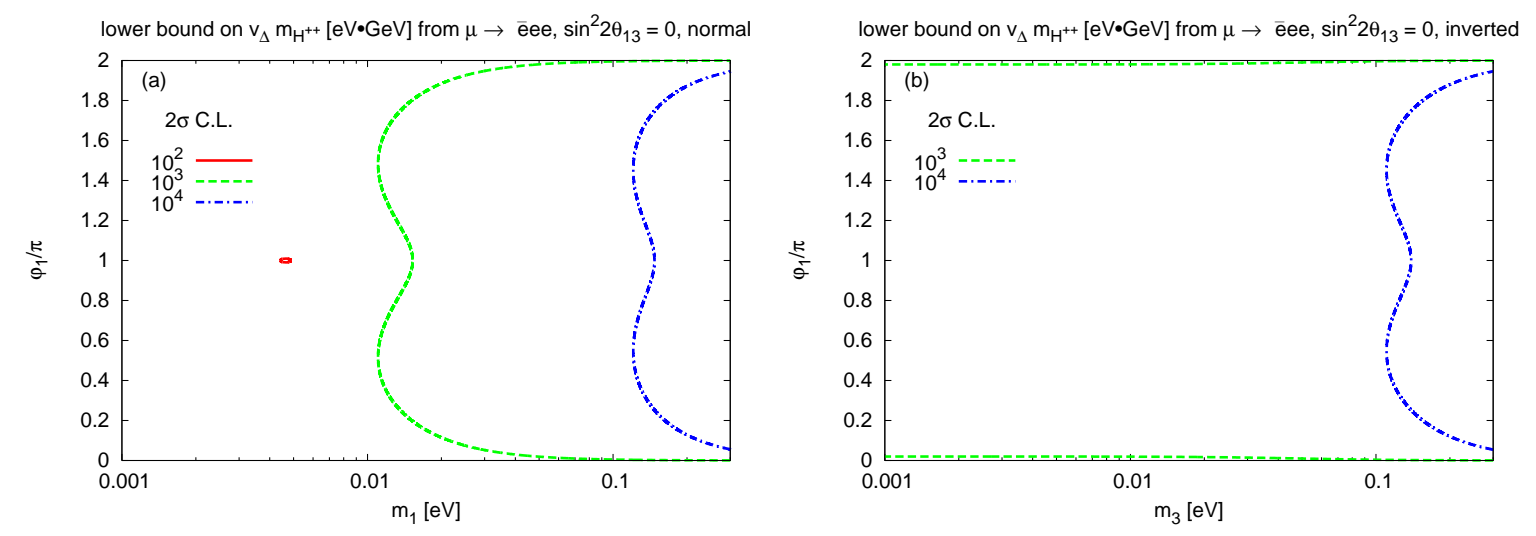

FIG. 2: Contours of the lower bounds on $v_{\Delta} m_{H^{ \pm \pm}}[\mathrm{eV} \cdot \mathrm{GeV}]$ given by $\mu \rightarrow \bar{e} e e$. (a) for the normal mass ordering. (b) for the inverted mass ordering.
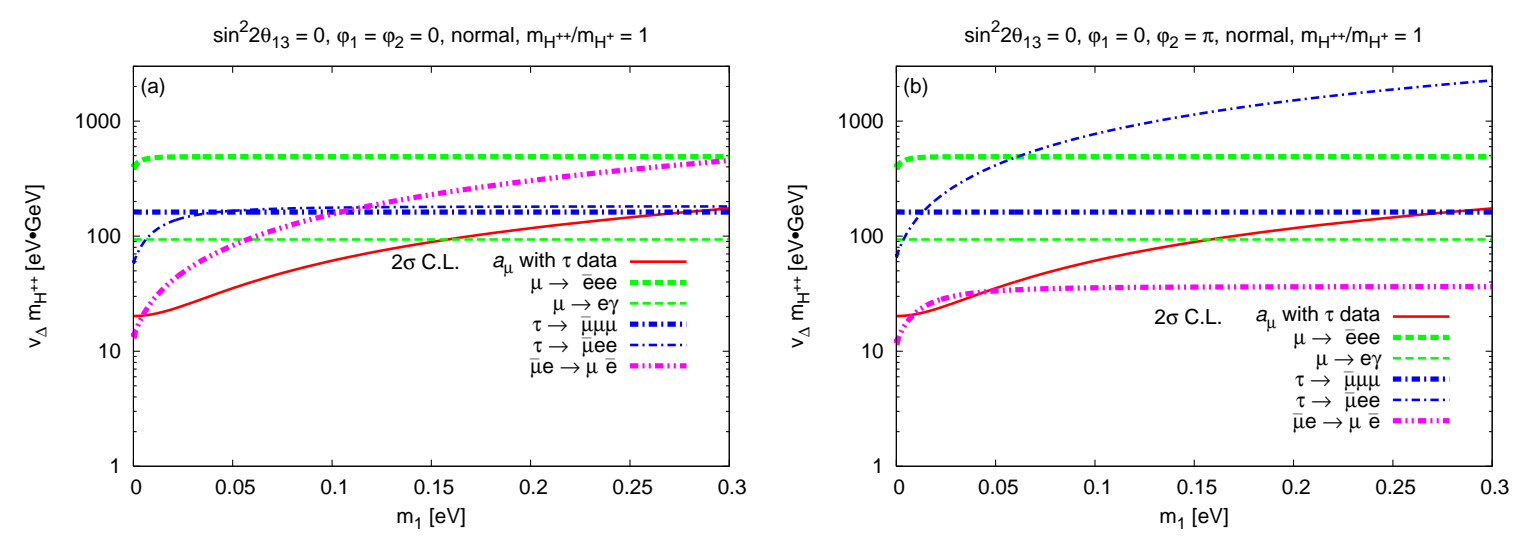

FIG. 3: Lower bounds on $v_{\Delta} m_{H^{ \pm \pm}}$for $\varphi_{1}=0$ in the normal mass ordering. (a) for $\varphi_{2}=0$. (b) for $\varphi_{2}=\pi$.

\section{B. Constraints in the case of $\mathbf{B R}(\mu \rightarrow \bar{e} e e) \neq 0$}

In most of parameter space, the strongest lower bound on $v_{\Delta} m_{H^{ \pm \pm}}$is given by $\mu \rightarrow \bar{e} e e$ as expected naively from the strong constraint on its branching ratio (20) $)$. Figures 2 (a) and (b) show contours of the bounds with $\theta_{13}=0$ for the normal and inverted mass ordering, respectively. Note that $\operatorname{BR}(\mu \rightarrow \bar{e} e e)$ does not depend on $\delta$ and $\varphi_{2}$ for $\theta_{13}=0$. Although the bound on $v_{\Delta} m_{H^{ \pm \pm}}$from $\mu \rightarrow \bar{e} e e$ is relatively weak for small $m_{1}$ in Fig. 2(a), bounds from other LFV processes are weaker than that. It is shown also that $\varphi_{1} \simeq 0$ makes the bound from $\mu \rightarrow \bar{e} e e$ weak for both of mass orderings. We focus on the case of $\varphi_{1}=0$ in the next 


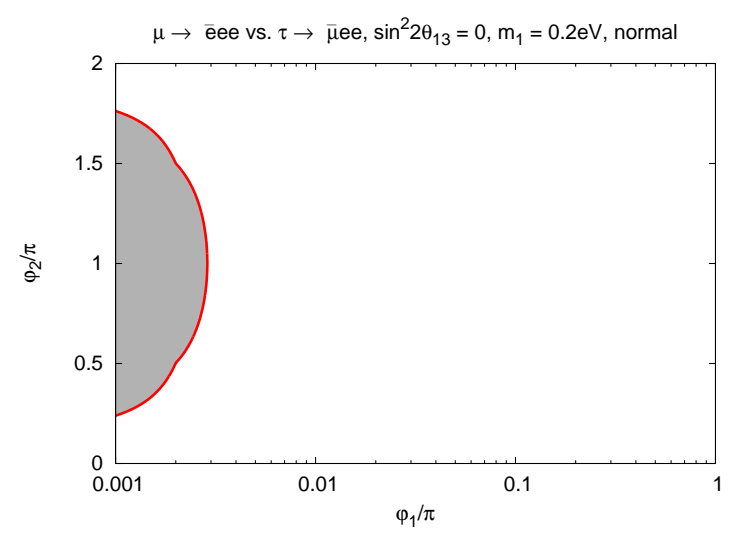

FIG. 4: In the shaded region, the bound on $v_{\Delta} m_{H^{ \pm \pm}}$from $\tau \rightarrow \bar{\mu} e e$ is stronger than the one from $\mu \rightarrow \bar{e} e e$. We used $m_{1}=0.2 \mathrm{eV}$ for the normal mass ordering. The shaded region is symmetric under a transformation of $\left(\varphi_{1}, \varphi_{2}-\pi\right) \rightarrow\left(-\varphi_{1},-\varphi_{2}+\pi\right)$.

paragraph. In Fig. 2(a) there is a special point at $\varphi_{1}=\pi$ and $m_{1}=s_{12}^{2} \sqrt{\Delta m_{21}^{2}} / \sqrt{\cos 2 \theta_{12}} \simeq$ $4.6 \times 10^{-3} \mathrm{eV}$ where the bound vanishes because of $m_{e e}=0$. Such cases of $\operatorname{BR}(\mu \rightarrow \bar{e} e e)=0$ are discussed in the next subsection.

In Fig. 3, $m_{1}$-dependences of bounds from $\Delta a_{\mu}[\tau]$ and LFV processes are presented for the normal mass ordering at $\varphi_{1}=0$ where the bound from $\mu \rightarrow \bar{e} e e$ is relatively weak. Figures 3(a) and (b) are obtained for $\varphi_{2}=0$ and $\pi$, respectively. Other parameters are the same values as ones in Fig. 1. It is seen in Fig. 3(a) that $\mu \rightarrow \bar{e} e e$ still gives the most stringent bound for $\varphi_{1}=\varphi_{2}=0$ and $m_{1} \lesssim 0.3 \mathrm{eV}$ although the bound from the muonium conversion gets close to that for large $m_{1}$. If we accept $m_{1} \gtrsim 0.3 \mathrm{eV}$, the bound from the muonium conversion can be stronger than the bound from $\mu \rightarrow \bar{e} e e$. On the other hand, Fig. 3(b) shows that the bound from $\tau \rightarrow \bar{\mu} e e$ can be more stringent than the one from $\mu \rightarrow \bar{e} e e$ for $m_{1} \gtrsim 0.06 \mathrm{eV}$. This is because a parameter set $\left(\theta_{13}, \varphi_{1}, \varphi_{2}\right)=(0,0, \pi)$ in the region of $\Delta m_{i j}^{2} / m_{1}^{2} \ll 1$ gives

$$
\begin{aligned}
\left|m_{\mu e}\right|^{2}\left|m_{e e}\right|^{2} & \simeq \frac{1}{32}\left(\Delta m_{21}^{2}\right)^{2} \sin ^{2} 2 \theta_{12} \simeq 1.6 \times 10^{-10} \mathrm{eV}^{4}, \\
\left|m_{\tau \mu}\right|^{2}\left|m_{e e}\right|^{2} & \simeq m_{1}^{4}
\end{aligned}
$$

and the large difference between experimental constraints (20) and (23) can be compensated for $m_{1} \gtrsim O(0.1) \mathrm{eV}$. In Fig. 4 the shaded region shows values of Majorana phases for which the bound from $\tau \rightarrow \bar{\mu} e e$ becomes more stringent than the one from $\mu \rightarrow \bar{e} e e$ at 
$m_{1}=0.2 \mathrm{eV}$ for the normal mass ordering. The region is symmetric under a transformation of $\left(\varphi_{1}, \varphi_{2}-\pi\right) \rightarrow\left(-\varphi_{1},-\varphi_{2}+\pi\right)$ because of $\left|m_{\ell \ell^{\prime}}\right|=\left|m_{\ell \ell^{\prime}}^{*}\right|$. Although the bound from $\mu \rightarrow \bar{e} e e$ is relatively weak for $\varphi_{1} \simeq 0$, the bound is still the most stringent one at around $\varphi_{2}=0$ because $\tau \rightarrow \bar{\mu} e e$ is also suppressed. If we take nonzero $\theta_{13}$ and ignore $\Delta m_{21}^{2}$ for $\left(\varphi_{1}, \varphi_{2}\right)=(0, \pi)$, eq. (26) is rewritten as

$$
\left|m_{\mu e}\right|^{2}\left|m_{e e}\right|^{2} \simeq 2 s_{13}^{2} m_{1}^{4}
$$

while eq. (27) remains valid. Therefore, the shaded region in Fig. 团 at around $\left(\varphi_{1}, \varphi_{2}\right)=$ $(0, \pi)$ exists for $\sin ^{2} 2 \theta_{13} \lesssim 10^{-5}$. For the inverted mass ordering, the region where $\tau \rightarrow \bar{\mu} e e$ becomes remarkable is almost same as the one in Figs. 3 and 4 because neutrino masses are almost degenerated in the region. In such a region, we can also expect a signal of $\tau \rightarrow \bar{\mu} e e$ in future experiments [35, 36] with satisfying the current constraint on $\mu \rightarrow \bar{e} e e$.

\section{Constraints in Cases of $\mathbf{B R}(\mu \rightarrow \bar{e} e e)=0$}

It has been known that the strong constraint from $\mu \rightarrow \bar{e} e e$ can be evaded in the cases of $m_{e \mu}=0$ [14] and $m_{e e}=0$ [6]. While it is impossible to have $m_{e \mu}=0$ with $\theta_{13}=0$, the case of $m_{e e}=0$ is possible also for $\theta_{13}=0$ as we mentioned for Fig. 2(a). Such cancellations in the HTM are desired also for experiments [35, 36, 37] to discover some LFV decays ( $\mu \rightarrow e \gamma$ etc.) [6, 14] in the future. Figures 5(a)-(d) show results for the case of $m_{e \mu}=0$ in the normal mass ordering. Four CP conserving sets of Majorana phases are taken for the figures as examples. We use appropriate values of $\theta_{13}$ and $\delta$ for $m_{e \mu}=0$, which we call as "magic values" $\theta_{13}^{\mathrm{mgc}}$ and $\delta^{\mathrm{mgc}}$, and explicit formulae of them are shown in Appendix. For

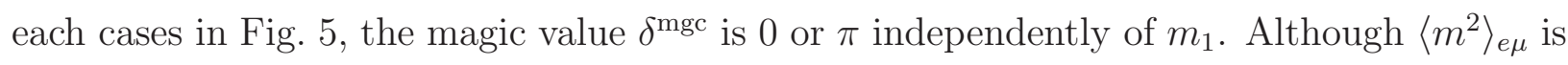
independent of $m_{1}$ and Majorana phases, the bounds from $\mu \rightarrow e \gamma$ in Fig. [5 are not constant with respect to these parameters because $\theta_{13}^{\mathrm{mgc}}$ depends on them. We see in Figs. 5 (a)-(d) that the bound from $\mu \rightarrow e \gamma$ is the strongest one for $m_{1} \simeq 0$, and this is also the case with any values of $\varphi_{1}$ and $\varphi_{2}$. For the case of Fig 5 (a), $\tau$ decays give the most stringent bound for $0.01 \mathrm{eV} \lesssim m_{1} \lesssim 0.12 \mathrm{eV}$, and the bound from the muonium conversion becomes the strongest one for $m_{1} \gtrsim 0.12 \mathrm{eV}$. In Fig. 5 (b), the bound from $\tau \rightarrow \bar{\mu} e e$ is prominent. The

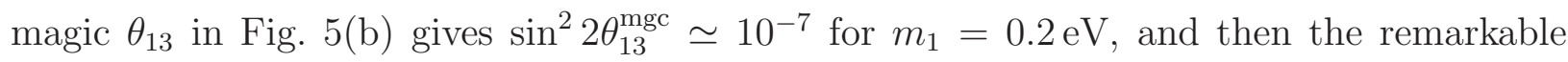
behavior of the bound from $\tau \rightarrow \bar{\mu} e e$ can be understood also as a part of the case shown in 

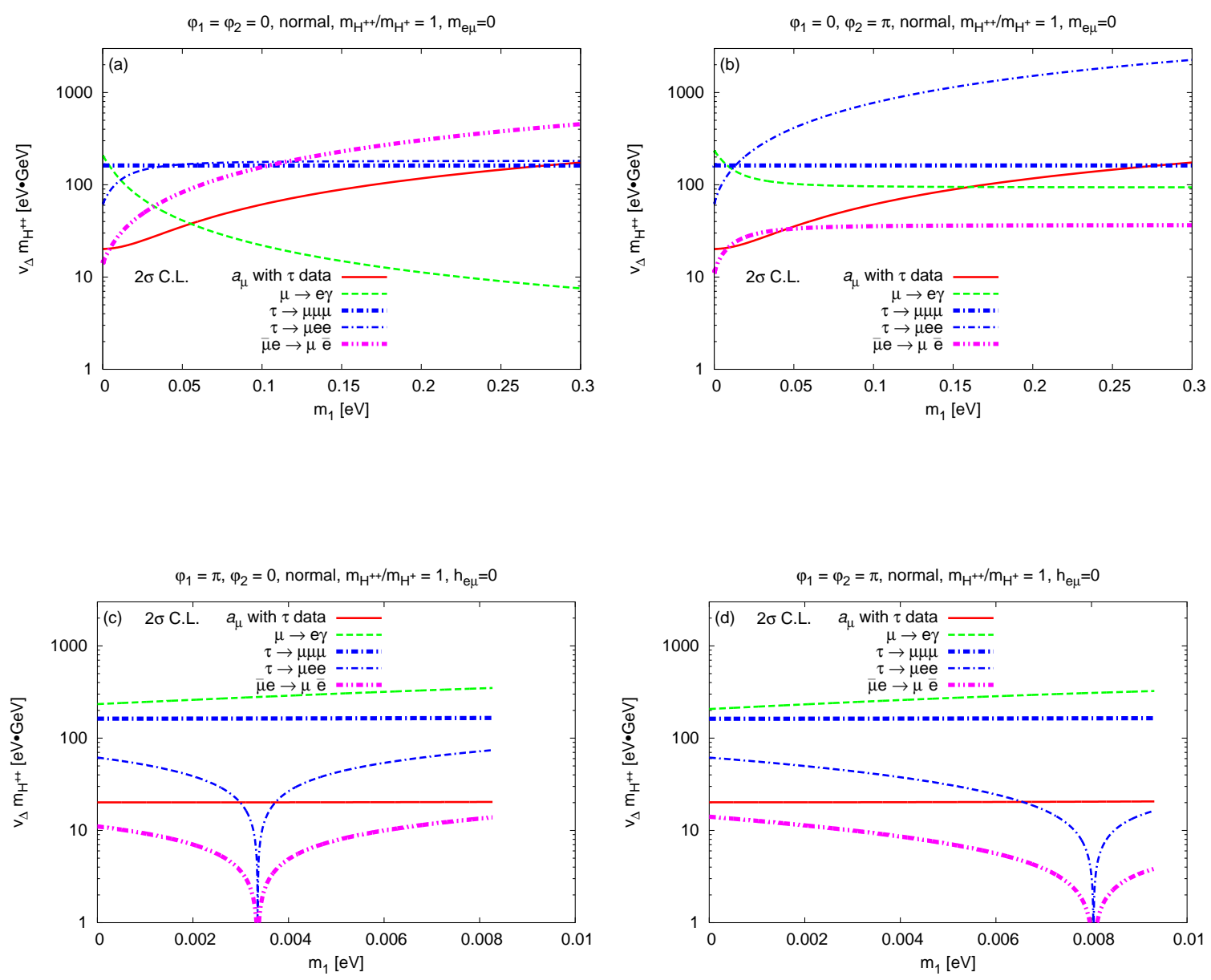

FIG. 5: Lower bounds on $v_{\Delta} m_{H^{ \pm \pm}}$for cases of $m_{e \mu}=0$ in the normal mass ordering. The value of $\theta_{13}^{\mathrm{mgc}}$ varies to keep $m_{e \mu}=0$ (See Appendix). (a) for $\left(\varphi_{1}, \varphi_{2}\right)=(0,0), \delta^{\mathrm{mgc}}=\pi$. (b) for $\left(\varphi_{1}, \varphi_{2}\right)=(0, \pi), \delta^{\mathrm{mgc}}=0 .(\mathrm{c})$ for $\left(\varphi_{1}, \varphi_{2}\right)=(\pi, 0), \delta^{\mathrm{mgc}}=0 .(\mathrm{d})$ for $\left(\varphi_{1}, \varphi_{2}\right)=(\pi, \pi), \delta^{\mathrm{mgc}}=\pi$.

Fig. 4 whose shaded region appears for $\sin ^{2} 2 \theta_{13} \lesssim 10^{-5}$. In Figs. 5(c) and (d), the most stringent bound is obtained from $\mu \rightarrow e \gamma$. Note that $\sin ^{2} 2 \theta_{13}^{\mathrm{mgc}}$ in Figs. 5(c) and (d) become larger than 0.14 of the CHOOZ bound [10] for $m_{1} \gtrsim 0.008 \mathrm{eV}$, and then we can not have $m_{e \mu}=0$ for the case.

Figure 6] shows which process gives the most stringent lower bound on $v_{\Delta} m_{H^{ \pm \pm}}$in a space of Majorana phases by keeping $m_{e \mu}=0$ for the normal mass ordering. Green circles, blue crosses, and magenta triangles show regions where the strongest bound comes from $\mu \rightarrow e \gamma, \tau \rightarrow \bar{\mu} e e$, and the muonium conversion, respectively. It is impossible to achieve $m_{e \mu}=0$ outside of the regions because of unacceptably large $\theta_{13}^{\mathrm{mgc}}$, and then it becomes 

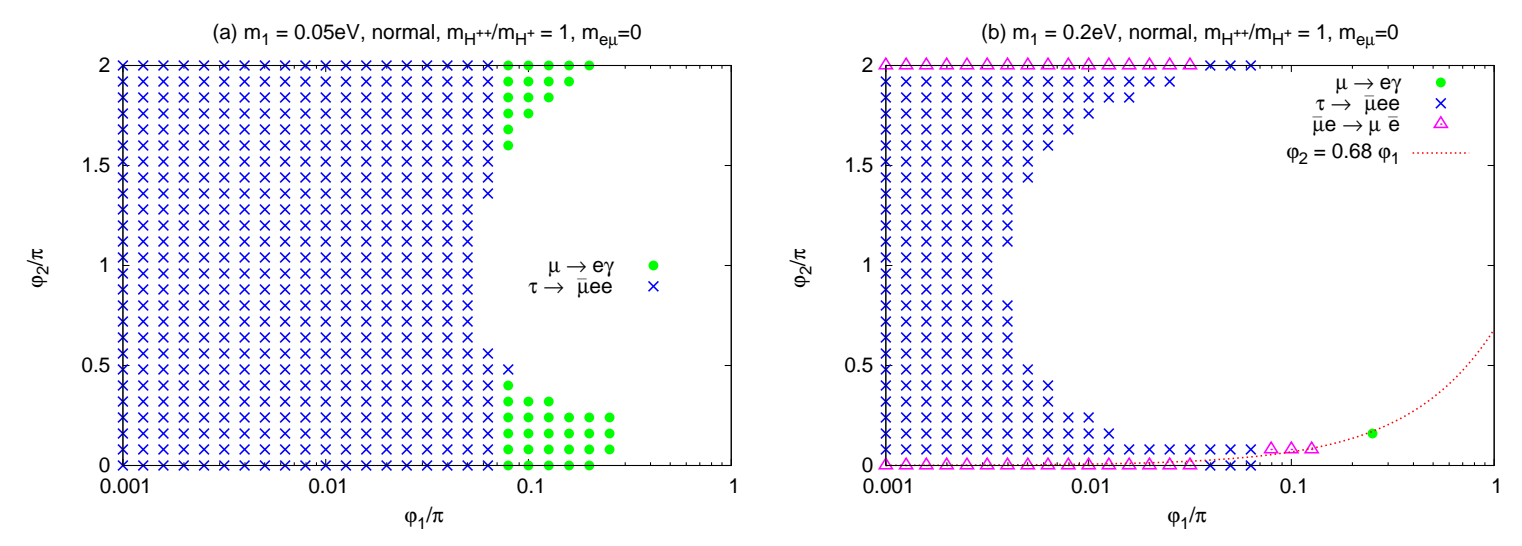

FIG. 6: Green circles, blue crosses, and magenta triangles show regions where the strongest lower bound on $v_{\Delta} m_{H^{ \pm \pm}}$for the case of $m_{e \mu}=0$ in the normal mass ordering comes from $\mu \rightarrow e \gamma$, $\tau \rightarrow \bar{\mu} e e$, and the muonium conversion, respectively. The values of $\theta_{13}^{\mathrm{mgc}}$ and $\delta^{\mathrm{mgc}}$ are functions of Majorana phases (See Appendix). We can not have $m_{e \mu}=0$ outside of these regions. The regions are symmetric under a transformation $\left(\varphi_{1}, \varphi_{2}\right) \rightarrow\left(-\varphi_{1},-\varphi_{2}\right)$. (a) with $m_{1}=0.05 \mathrm{eV}$. (b) with $m_{1}=0.2 \mathrm{eV}$. With $m_{1}=0$, the most stringent bound is given by $\mu \rightarrow e \gamma$ for all values of $\varphi_{1}$ and $\varphi_{2}$

the situations discussed in the previous subsection for $\operatorname{BR}(\mu \rightarrow \bar{e} e e) \neq 0$. Figure 6 (a) is for $m_{1}=0.05 \mathrm{eV}$ and (b) is for $m_{1}=0.2 \mathrm{eV}$. For $m_{1}=0$, it is possible to have $m_{e \mu}=0$ for any values of Majorana phases, and the most stringent bound is always given by $\mu \rightarrow e \gamma$ as $v_{\Delta} m_{H^{ \pm \pm}} \gtrsim 200 \mathrm{eV} \cdot \mathrm{GeV}$. Values of lower bounds on $v_{\Delta} m_{H^{ \pm \pm}}$for Fig. 6(a) from $\mu \rightarrow e \gamma$ and $\tau \rightarrow \bar{\mu} e e$ vary in $150 \rightarrow 350 \mathrm{eV} \cdot \mathrm{GeV}$ and $150 \rightarrow 400 \mathrm{eV} \cdot \mathrm{GeV}$, respectively. We have $v_{\Delta} m_{H^{ \pm \pm}} \gtrsim 300 \mathrm{eV} \cdot \mathrm{GeV}, \gtrsim 300 \rightarrow 1500 \mathrm{eV} \cdot \mathrm{GeV}$, and $\gtrsim 300 \mathrm{eV} \cdot \mathrm{GeV}$ in Fig. 6(b) from $\mu \rightarrow e \gamma, \tau \rightarrow \bar{\mu} e e$, and the muonium conversion, respectively. The regions are symmetric under a transformation $\left(\varphi_{1}, \varphi_{2}\right) \rightarrow\left(-\varphi_{1},-\varphi_{2}\right)$ because of $\left|m_{\ell \ell^{\prime}}\right|=\left|m_{\ell \ell^{\prime}}^{*}\right|$. We see that $\varphi_{1} \simeq 0$ is preferred to keep $s_{13}^{\mathrm{mgc}}$ small for $m_{1} \neq 0$ and we can confirm $s_{13}^{\mathrm{mgc}} \propto \Delta m_{21}^{2}$ for $\varphi_{1}=0$ with eq. (32) in Appendix. It can be found also with eq. (32) for $\varphi_{1}, \varphi_{2} \ll 1$ and $\Delta m_{i j}^{2}=0$ that

$$
\left.\varphi_{2} \simeq \frac{1+\cos 2 \theta_{12}}{2} \varphi_{1}=0.68 \varphi_{1} \quad \text { (red dotted line in Fig. 6 }(\mathrm{b})\right)
$$

is preferred to have a small $s_{13}^{\mathrm{mgc}}$. Bounds from $\tau \rightarrow \bar{\mu} e e$ and the muonium conversion can be the most stringent one only for $\varphi_{1} \lesssim 0.1 \pi$. Majorana phases are almost restricted as 

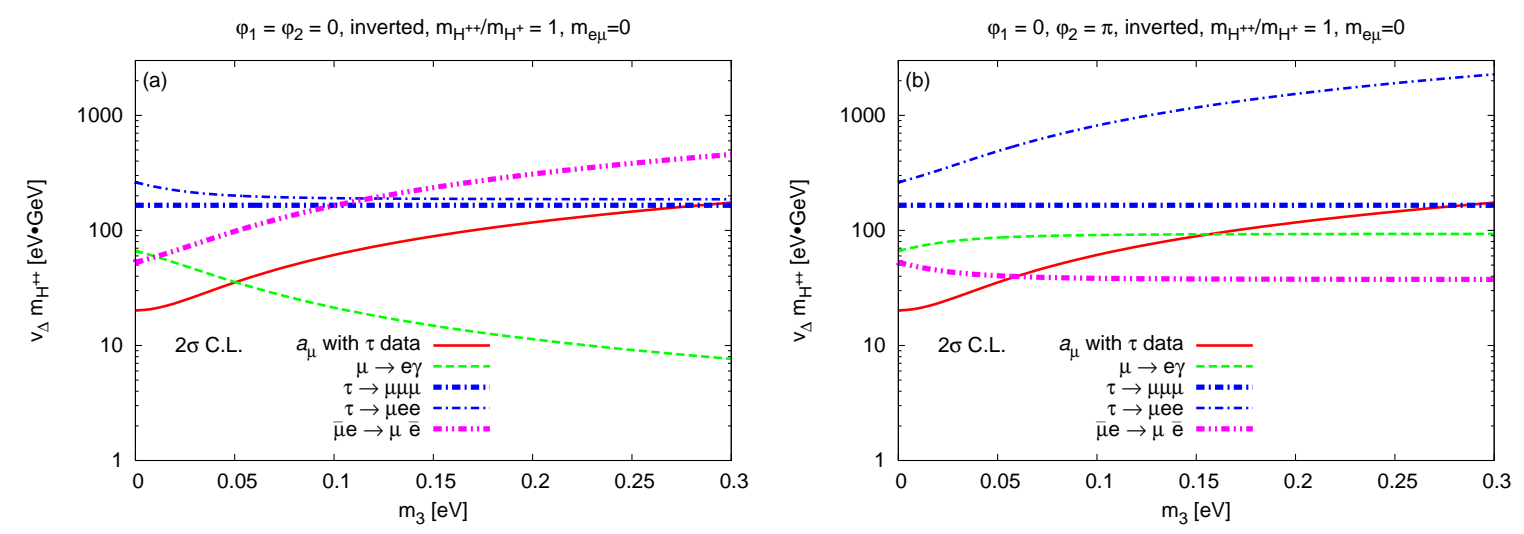

FIG. 7: Lower bounds on $v_{\Delta} m_{H^{ \pm \pm}}$for cases of $m_{e \mu}=0$ in the inverted mass ordering. The value of $\theta_{13}^{\mathrm{mgc}}$ varies to keep $m_{e \mu}=0$ (See Appendix). (a) for $\left(\varphi_{1}, \varphi_{2}\right)=(0,0), \delta^{\mathrm{mgc}}=0$. (b) for $\left(\varphi_{1}, \varphi_{2}\right)=(0, \pi), \delta^{\mathrm{mgc}}=0$.

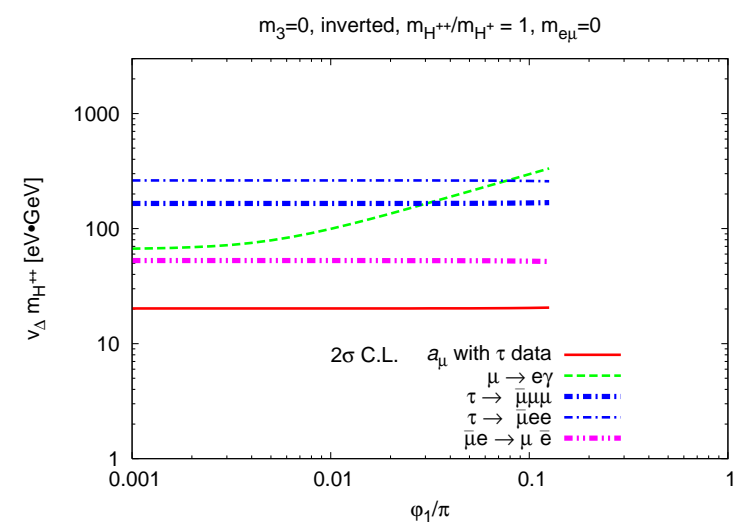

FIG. 8: The $\varphi_{1}$-dependence of lower bounds on $v_{\Delta} m_{H^{ \pm \pm}}$for cases of $m_{e \mu}=0$ with $m_{3}=0$ in the inverted mass ordering. $\theta_{13}^{\mathrm{mgc}}$ and $\delta^{\mathrm{mgc}}$ for $m_{e \mu}=0$ are functions of $\varphi_{1}$ (See Appendix).

$\varphi_{2} \simeq 0.68 \varphi_{1}$ for the case of a strong constraint from the muonium conversion. It is shown that $\mu \rightarrow e \gamma$ can be the most stringent bound even for $m_{1} \gtrsim 0.008 \mathrm{eV}$ (cf. Fig. [5) because of large $s_{13}^{\mathrm{mgc}}$. At the border to the white region in Fig. 6, we have $\sin ^{2} 2 \theta_{13}^{\mathrm{mgc}}=0.14$.

Similarly to Fig. [5, lower bounds on $v_{\Delta} m_{H^{ \pm \pm}}$are shown in Fig. 7 for the inverted mass ordering. Note that $\varphi_{1}=\pi$ can not give $m_{e \mu}=0$ for the mass ordering because $s_{13}^{\text {mgc }}$ becomes too large. Very roughly speaking, the results in Figs. 7(a) and (b) are the same as those in Figs. 5(a) and (b), respectively. A difference is that $\tau \rightarrow \bar{\mu} e e$ can be the most stringent 


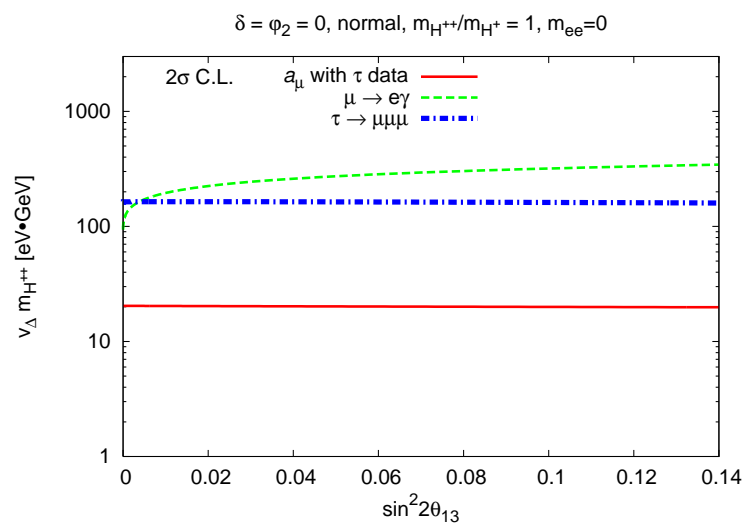

FIG. 9: Lower bounds on $v_{\Delta} m_{H^{ \pm \pm}}$for cases of $m_{e e}=0$ with $\delta=\varphi_{2}=0$ in the normal mass ordering. Values of $m_{1}^{\mathrm{mgc}}$ and $\varphi_{1}^{\mathrm{mgc}}$ are given by the condition $m_{e e}=0$ depending on $\theta_{13}$ (See Appendix).

bound at $m_{3}=0$ while it is not the case for $m_{1}=0$ in the normal mass ordering. Figure 8 shows the $\varphi_{1}$-dependence for $m_{e \mu}=0$ with $m_{3}=0$ where the $\varphi_{2}$-dependence vanishes. For $\varphi_{1} \gtrsim 0.1 \pi, \sin ^{2} 2 \theta_{13}^{\mathrm{mgc}}$ becomes larger than 0.14 . We see that $\mu \rightarrow e \gamma$ can give the most stringent bound even for the inverted mass ordering. For $m_{3}=0.2 \mathrm{eV}$, Fig. 6(b) is almost applicable to see which process gives the most stringent bound.

Figure 9 is the result for the case of $m_{e e}=0$ which is possible only in the normal mass ordering. Formulae of the magic values $m_{1}^{\mathrm{mgc}}$ and $\varphi_{1}^{\mathrm{mgc}}$ for $m_{e e}=0$ are shown in Appendix. The bound from $\mu \rightarrow e \gamma$ is the most stringent one except for $\sin ^{2} 2 \theta_{13} \lesssim 0.04$ where the bound from $\tau \rightarrow \bar{\mu} \mu \mu$ becomes stronger than that. This is also the case for different values of $\delta$ and $\varphi_{2}$.

\section{CONCLUSION}

In the HTM, it is impossible to have a contribution to the muon anomalous MDM with a plus sign. Therefore, the HTM is qualitatively disfavored by positive $\Delta a_{\mu}[\tau]\left(\Delta a_{\mu}\left[e^{+} e^{-}\right]\right)$ at $1.8 \sigma(3.7 \sigma)$. If we deal with $2 \sigma$ bounds to avoid the disagreement with $\Delta a_{\mu}[\tau], v_{\Delta} m_{H^{ \pm \pm}}$ must be greater than $10^{3} \mathrm{eV} \cdot \mathrm{GeV}$ in most of parameter space of the HTM in order to satisfy a strong constraint on $\operatorname{BR}(\mu \rightarrow \bar{e} e e)$. We found that the bound on $v_{\Delta} m_{H^{ \pm \pm}}$from $\tau \rightarrow \bar{\mu} e e$ becomes more stringent than that from $\mu \rightarrow \bar{e} e e$ in a region of $m_{1} \gtrsim 0.06 \mathrm{eV}, \varphi_{1} \lesssim 0.002 \pi$, 
$0.5 \pi \lesssim \varphi_{2} \lesssim 1.5 \pi$, and $\sin ^{2} 2 \theta_{13} \lesssim 10^{-5}$. The bound from $\mu \rightarrow \bar{e} e e$ can be evaded in cases of $m_{e \mu}=0$ [14] and $m_{e e}=0$ [6]. In the case of $m_{e \mu}=0$ in the normal mass ordering, the strongest bound is given by $\mu \rightarrow e \gamma$ for $m_{1} \lesssim 0.01 \mathrm{eV}$ or $\varphi_{1} \gtrsim 0.1 \pi$ and by $\tau$ decays (mainly $\tau \rightarrow \bar{\mu} e e)$ for $m_{1} \gtrsim 0.01 \mathrm{eV}$ with $\varphi_{1} \lesssim 0.1 \pi$. On the other hand, $\tau \rightarrow \bar{\mu} e e$ gives the strongest bound in the normal mass ordering except for $m_{3} \simeq 0$ with $\varphi_{1} \simeq 0.1 \pi$ where $\mu \rightarrow e \gamma$ gives the bound. For both of the mass orderings with $m_{e \mu}=0$, the muonium conversion gives the most stringent bound if Majorana phases satisfy $\varphi_{2} \simeq 0.68 \varphi_{1}$ for $\varphi_{1}, \varphi_{2} \ll 1$ and $m_{1} \gtrsim 0.1 \mathrm{eV}$. In the case of $m_{e e}=0$, the strongest bound is obtained from $\mu \rightarrow e \gamma$ except for the case of $\sin ^{2} 2 \theta_{13} \lesssim 0.04$ where the bound is given by $\tau \rightarrow \bar{\mu} \mu \mu$. By looking over all cases, we see that $v_{\Delta} m_{H^{ \pm \pm}} \gtrsim 150 \mathrm{eV} \cdot \mathrm{GeV}$ should be satisfied in the HTM. If $m_{H \pm \pm}$ is measured, the bound can be the lower bound on $v_{\Delta}$ though there remains a possibility of $v_{\Delta}=0$ for which we can not use the correlation of $h_{\ell \ell^{\prime}}$ with $\sqrt{2} v_{\Delta} h_{\ell \ell^{\prime}}=m_{\ell \ell^{\prime}}$.

\section{Acknowledgements}

We thank A.G. Akeroyd for useful discussions. The work of T. F. is supported in part by the Grant-in-Aid for Scientific Research from the Ministry of Education, Science and Culture of Japan (No. 20540282).

\section{APPENDIX}

We obtained formulae of the magic values of $\theta_{13}$ and $\delta$, which give $m_{e \mu}=0$, as

$$
\begin{aligned}
& \cos \delta^{\mathrm{mgc}}=-\frac{c_{12} s_{12} c_{23} C}{s_{23} s_{13}^{\mathrm{mgc}}\left\{m_{3}^{2}-s_{12}^{4} m_{2}^{2} \sin ^{2} \varphi_{1}-\left(s_{12}^{2} m_{2} \cos \varphi_{1}+c_{12}^{2} m_{1}\right)^{2}\right\}}, \\
& \sin \delta^{\mathrm{mgc}}=\frac{c_{12} s_{12} c_{23} D}{s_{23} s_{13}^{\mathrm{mgc}}\left\{m_{3}^{2}-s_{12}^{4} m_{2}^{2} \sin ^{2} \varphi_{1}-\left(s_{12}^{2} m_{2} \cos \varphi_{1}+c_{12}^{2} m_{1}\right)^{2}\right\}} \\
& s_{13}^{\mathrm{mgc}}=\frac{c_{12} s_{12} c_{23} \sqrt{C^{2}+D^{2}}}{s_{23}\left|m_{3}^{2}-s_{12}^{4} m_{2}^{2} \sin ^{2} \varphi_{1}-\left(s_{12}^{2} m_{2} \cos \varphi_{1}+c_{12}^{2} m_{1}\right)^{2}\right|} \\
& C \equiv-c_{12}^{2} m_{1}^{2}+s_{12}^{2} m_{2}^{2}+m_{1} m_{2} \cos 2 \theta_{12} \cos \varphi_{1} \\
&-m_{1} m_{3} \cos \varphi_{2}+m_{2} m_{3} \cos \left(\varphi_{1}-\varphi_{2}\right), \\
& D \\
& \equiv-m_{1} m_{2} \sin \varphi_{1}+m_{1} m_{3} \sin \varphi_{2}+m_{2} m_{3} \sin \left(\varphi_{1}-\varphi_{2}\right),
\end{aligned}
$$


where we define $s_{13}^{\mathrm{mgc}} \equiv \sin \theta_{13}^{\mathrm{mgc}}$. Note that $s_{13}^{\mathrm{mgc}} \neq 0$ because it requires $m_{2}=m_{1}$. Note also that $s_{13}^{\mathrm{mgc}}$ is not always acceptable; For example, $\varphi_{1}=\pi$ in the inverted mass ordering gives $s_{13}^{\mathrm{mgc}}>1$. These results are consistent with $s_{13}^{\mathrm{mgc}}$ and $\delta^{\mathrm{mgc}}$ used in [6, 14] for $\varphi_{1}, \varphi_{2}=0$ or $\pi$. Although mixing matrix in [6] is defined as $\nu_{\ell}=\sum_{i} U_{\ell i}^{*} \nu_{i}$ in stead of $\nu_{\ell}=\sum_{i} U_{\ell i} \nu_{i}$ used in this article, there is no change in formulae of magic values because the difference appears just as the simultaneous flip of signs of all phases.

On the other hand, $m_{e e}=0$ can be achieved only in the normal mass ordering. The magic values of $\varphi_{1}$ and $m_{1}$ for $m_{e e}=0$ are given as functions of $s_{13}$ and $\varphi_{2}-2 \delta$ [6] by

$$
\begin{aligned}
\sin \varphi_{1}^{\mathrm{mgc}} \equiv & -\frac{\sqrt{\left(m_{1}^{\mathrm{mgc}}\right)^{2}+\Delta m_{31}^{2}}}{s_{12}^{2} \sqrt{\left(m_{1}^{\mathrm{mgc}}\right)^{2}+\Delta m_{21}^{2}}} t_{13}^{2} \sin \left(\varphi_{2}-2 \delta\right), \quad \cos \varphi_{1}^{\mathrm{mgc}} \leq 0 \\
\left(m_{1}^{\mathrm{mgc}}\right)^{2} \equiv & \frac{1}{\cos ^{2} 2 \theta_{12}-2\left(s_{12}^{4}+c_{12}^{4} \cos 2\left(\varphi_{2}-2 \delta\right)\right) t_{13}^{4}+t_{13}^{8}} \\
& \times\left[s_{12}^{4} \cos 2 \theta_{12} \Delta m_{21}^{2}+\left\{s_{12}^{4} \Delta m_{21}^{2}+\left(s_{12}^{4}+c_{12}^{4} \cos 2\left(\varphi_{2}-2 \delta\right)\right) \Delta m_{31}^{2}\right\} t_{13}^{4}\right. \\
& \left.-\Delta m_{31}^{2} t_{13}^{8}-2 c_{12}^{2} t_{13}^{2} \cos \left(\varphi_{2}-2 \delta\right) \sqrt{A+B t_{13}^{4}}\right], \\
A \equiv & \left(s_{12}^{4} \Delta m_{21}^{2}+\cos 2 \theta_{12} \Delta m_{31}^{2}\right) s_{12}^{4} \Delta m_{21}^{2}, \\
B \equiv & \left\{\left(s_{12}^{4}-c_{12}^{4} \sin ^{2}\left(\varphi_{2}-2 \delta\right)\right) \Delta m_{31}^{2}-s_{12}^{4} \Delta m_{21}^{2}\right\} \Delta m_{31}^{2},
\end{aligned}
$$

where we define $t_{13} \equiv s_{13} / c_{13}$.

[1] W. Konetschny and W. Kummer, Phys. Lett. B 70, 433 (1977); T. P. Cheng and L. F. Li, Phys. Rev. D22, 2860 (1980).

[2] J. Schechter and J. W. F. Valle, Phys. Rev. D 22, 2227 (1980).

[3] C. Amsler et al. [Particle Data Group], Phys. Lett. B 667, 1 (2008).

[4] S. M. Bilenky, J. Hosek and S. T. Petcov, Phys. Lett. B 94, 495 (1980); M. Doi, T. Kotani, H. Nishiura, K. Okuda and E. Takasugi, Phys. Lett. B 102, 323 (1981).

[5] Z. Maki, M. Nakagawa and S. Sakata, Prog. Theor. Phys. 28, 870 (1962).

[6] A. G. Akeroyd, M. Aoki and H. Sugiyama, Phys. Rev. D 79, 113010 (2009).

[7] B. T. Cleveland et al., Astrophys. J. 496, 505 (1998); W. Hampel et al. [GALLEX Collaboration], Phys. Lett. B 447, 127 (1999); J. N. Abdurashitov et al. [SAGE Collaboration], J. Exp. Theor. Phys. 95, 181 (2002) [Zh. Eksp. Teor. Fiz. 122, 211 (2002)] arXiv:astro-ph/0204245]; 
J. Hosaka et al. [Super-Kamkiokande Collaboration], Phys. Rev. D 73, 112001 (2006); B. Aharmim et al. [SNO Collaboration], Phys. Rev. Lett. 101, 111301 (2008); C. Arpesella et al. [The Borexino Collaboration], Phys. Rev. Lett. 101, 091302 (2008)

[8] Y. Ashie et al. [Super-Kamiokande Collaboration], Phys. Rev. D 71, 112005 (2005); J. L. Raaf [Super-Kamiokande Collaboration], J. Phys. Conf. Ser. 136, 022013 (2008).

[9] M. H. Ahn et al. [K2K Collaboration], Phys. Rev. D 74, 072003 (2006); P. Adamson et al. [MINOS Collaboration], Phys. Rev. Lett. 101, 131802 (2008)

[10] M. Apollonio et al. [CHOOZ Collaboration], Eur. Phys. J. C 27, 331 (2003).

[11] C. Kraus et al., Eur. Phys. J. C 40, 447 (2005).

[12] E. Komatsu et al. [WMAP Collaboration], Astrophys. J. Suppl. 180, 330 (2009); J. Dunkley et al. [WMAP Collaboration], Astrophys. J. Suppl. 180, 306 (2009).

[13] A. Abada, C. Biggio, F. Bonnet, M. B. Gavela and T. Hambye, JHEP 0712, 061 (2007).

[14] E. J. Chun, K. Y. Lee and S. C. Park, Phys. Lett. B 566, 142 (2003).

[15] M. Kakizaki, Y. Ogura and F. Shima, Phys. Lett. B 566, 210 (2003).

[16] G. W. Bennett et al. [Muon G-2 Collaboration], Phys. Rev. D 73, 072003 (2006).

[17] M. Davier et al., arXiv:0906.5443 [hep-ph].

[18] J. F. de Troconiz and F. J. Yndurain, Phys. Rev. D 71, 073008 (2005).

[19] K. Hagiwara, A. D. Martin, D. Nomura and T. Teubner, Phys. Lett. B 649, 173 (2007).

[20] M. Davier, S. Eidelman, A. Hocker and Z. Zhang, Eur. Phys. J. C 27, 497 (2003); Eur. Phys. J. C 31, 503 (2003); M. Davier, Nucl. Phys. Proc. Suppl. 169, 288 (2007).

[21] F. Jegerlehner, Acta Phys. Polon. B 38, 3021 (2007).

[22] J. Prades, arXiv:0909.2546 [hep-ph].

[23] J. P. Leveille, Nucl. Phys. B 137, 63 (1978); V. D. Barger, H. Baer, W. Y. Keung and R. J. N. Phillips, Phys. Rev. D 26, 218 (1982); J. F. Gunion, J. Grifols, A. Mendez, B. Kayser and F. I. Olness, Phys. Rev. D 40, 1546 (1989); R. M. Francis, M. Frank and C. S. Kalman, Phys. Rev. D 43, 2369 (1991); E. Accomando et al., arXiv:hep-ph/0608079 (Chapter 13).

[24] A. Dedes and H. E. Haber, JHEP 0105, 006 (2001).

[25] J. D. Bjorken and S. Weinberg, Phys. Rev. Lett. 38, 622 (1977); S. M. Barr and A. Zee, Phys. Rev. Lett. 65, 21 (1990) [Erratum-ibid. 65, 2920 (1990)].

[26] K. Cheung and O. C. W. Kong, Phys. Rev. D 68, 053003 (2003).

[27] K. Cheung, O. C. W. Kong and J. S. Lee, JHEP 0906, 020 (2009). 
[28] T. Moroi, Phys. Rev. D 53, 6565 (1996) [Erratum-ibid. D 56, 4424 (1997)].

[29] T. Fukuyama, T. Kikuchi and N. Okada, Phys. Rev. D 68, 033012 (2003).

[30] A. Zee, Nucl. Phys. B 264, 99 (1986); K. S. Babu, Phys. Lett. B 203, 132 (1988).

[31] U. Bellgardt et al. [SINDRUM Collaboration], Nucl. Phys. B 299, 1 (1988).

[32] M. L. Brooks et al. [MEGA Collaboration], Phys. Rev. Lett. 83, 1521 (1999).

[33] Y. Miyazaki et al. [Belle Collaboration], Phys. Lett. B 660, 154 (2008).

[34] L. Willmann et al., Phys. Rev. Lett. 82, 49 (1999).

[35] S. Hashimoto et al., "Letter of intent for KEK Super B Factory," KEK-REPORT-2004-4; A. G. Akeroyd et al. [SuperKEKB Physics Working Group], arXiv:hep-ex/0406071; M. Bona et al., arXiv:0709.0451 [hep-ex]; T. Browder, M. Ciuchini, T. Gershon, M. Hazumi, T. Hurth, Y. Okada and A. Stocchi, JHEP 0802, 110 (2008); T. E. Browder, T. Gershon, D. Pirjol, A. Soni and J. Zupan, arXiv:0802.3201 [hep-ph].

[36] R. Santinelli, eConf C0209101, WE14 (2002) [Nucl. Phys. Proc. Suppl. 123, 234 (2003)]; M. Giffels, J. Kallarackal, M. Kramer, B. O’Leary and A. Stahl, Phys. Rev. D 77, 073010 (2008).

[37] J. Adam et al. [MEG collaboration], arXiv:0908.2594 [hep-ex]. 